\title{
Gradient and Categorical Effects in Native and Non-native Nasal-rhotic Coordination
}

\author{
Alexei Kochetov, Laura Colantoni, and Jeffrey Steele \\ University of Toronto
}

\section{Introduction}

Languages are known to differ in their patterns of consonant-to-consonant coordination, which include the relative timing of consonant gestures and the processes of coarticulation and assimilation (Browman \& Goldstein, 1989; Gafos, 2002). Acquisition of a second language (L2) therefore involves learning these language-specific coordination patterns and the corresponding processes within and across words in the target language (Cebrian, 2000; Zsiga, 2003; Oh, 2008; Davidson, 2010). For example, in the English phrase in Rome, the two consonants across the word boundary /n\#. $/$ have inherently conflicting tongue tip targets: a closure at the alveolar ridge (and a lowering of the velum) for $/ \mathrm{n} /$ and a post-alveolar narrow opening (an approximant-like constriction) for $/ \mathrm{x} /$. In continuous speech, these articulatory gestures tend to overlap in time, resulting in gradient assimilation in constriction location and degree, that is, a partial, continuous retraction and opening of the tongue tip closure. This 'gestural blending' process is illustrated in Figure 1 using Articulatory Phonology representations (Browman \& Goldstein, 1989).

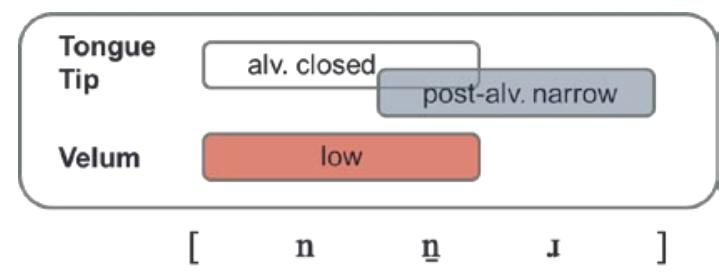

Figure 1. A gestural score for an /n/t/s/ sequence with two conflicting Tongue Tip gestures overlapping in time resulting in an [n]-to-[ㅁ] continuum during the nasal interval. The Tongue Tip gestures are specified for the Constriction Location ([alveolar] or [post-alveolar]) and the Constriction Degree ([closed] or [narrow]); the Velum gesture is specified for the Constriction Degree ([low]; see Browman \& Goldstein, 1989 on gestural representations).

In the present work, we seek to determine whether L2 learners of English with different L1 backgrounds acquire this pattern of gestural blending. Specifically, this involves learning both to appropriately produce the target sounds (an alveolar nasal $/ \mathrm{n} /$ in syllable coda and a post-alveolar approximant $/ \mathrm{I} /$ in onset) when they differ from the L1 and to temporally coordinate them with each other in a target-like sequence. In principle, there are several possible acquisition outcomes. First, learners might implement the $/ \mathrm{n} /+/ \mathrm{x} /$ sequence with no overlap, breaking up the sequence with an epenthetic vowel. Second, they might produce the sequence with a moderate degree of overlap and gradient assimilation (blending) as is typical for English (Barry, 1991; Stephenson \& Harrington, 2002). Third, they might implement the sequence with a large degree of overlap and categorical assimilation. Finally, if the realization of the sequence proves articulatorily too difficult, they might delete one of the two consonants, more likely the coda nasal. If assimilation is the chosen strategy, the process can involve place of articulation (constriction location), stricture (constriction degree) or both. Moreover, the process can be

\footnotetext{
* We thank the audience of AMP 2017 for insightful comments and suggestions. This work was supported by a University of Toronto Faculty of Arts \& Science ATLAS teaching grant to the authors and a Social Sciences and Humanities Research Council of Canada grant (\#435-2015-2013) to Alexei Kochetov.
} 
regressive, progressive or bi-directional. The strategy chosen in a particular case is likely to depend on L2 learners' native language and their English oral proficiency including the extent to which they have mastered the individual realization of the two phonemes.

To investigate how English $/ \mathrm{n} /+/ \mathrm{I} /$ sequences are realized by learners of different L1s, we collected electropalatographic (EPG) data from L2 learners who are native speakers of French, Japanese, and Spanish. These three languages differ in their phonetic realization of syllable-initial rhotics: French has a uvular fricative /в/ (e.g., Tranel, 1987), Spanish the alveolar trill /r/ (which is contrastive with a tap /r/, although not word-initially; Quilis, 1993), while Japanese has a (post-)alveolar flap / $/$ / (variably realized as a tap or a lateral; Vance, 1987). Alveolar nasals in word/utterance-final position are permitted in French (along with bilabial and palatal nasals; Tranel, 1987) and in many varieties of Spanish (e.g., Peninsular and Argentine). In other varieties of Spanish (e.g., Cuban), the default realization of final nasals is velar (Quilis, 1993). In Japanese, word-final nasals are realized as uvular approximants (Vance, 1987). Cross-word nasal + consonant sequences are known to show categorical patterns of coda nasal place assimilation in both Japanese (Vance, 1987; Stephenson \& Harrington, 2002; Kochetov, 2014) and Spanish (Quilis, 1993; Honorof, 1999; Kochetov \& Colantoni, 2010; Ramsammy, 2011) although the realization of nasal + rhotic sequences in this latter language is more variable compared to nasal + stop sequences (Kochetov, \& Colantoni, 2013). In contrast, nasal place assimilation in French is gradient, at least in $/ \mathrm{n} /+$ stop sequences (Steele, Colantoni, \& Kochetov, to appear).

On the assumption that L2 assimilation may be influenced by L1 articulatory patterns even at later stages of acquisition, we predict that Japanese and Spanish learners of English who fail to acquire English gradient patterns would produce categorical assimilation, even if the speakers learn to produce English-like nasals and rhotics. The distinction between the acquisition and transfer is less clear for French learners of English, given that L1 already exhibits gradient assimilation in L1, albeit to a lesser extent than in English.

\section{Method}

The female participants, who were residing in Toronto, Canada at the time of the experiment, included nine English learners, three native speakers each of French (FR1-3), Japanese (JP1-3), and Spanish (SP13). Two of the French speakers were from Quebec, the other from France. The Spanish speakers were born in Argentina, Cuba, and Spain. All three Japanese speakers came from the island of Honshu. Based on an English accentedness rating test, the participants' proficiency level was classified as either intermediate or advanced. Specifically, the accentedness scores (maximum score of 5.0) for the French speakers ranged from 1.9 to 4.4 (mean 3.0), from 1.9 to 2.7 (mean 2.3) for the Japanese speakers, and, for the Spanish speakers, from 2.0 to 3.0 (mean 2.4). In addition, we collected control data from three Canadian English speakers, one female and two males. Their accentedness scores ranged from 4.6 to 5.0 (mean 4.8).

The materials for the present analysis included the target utterance common ransom involving an $/ \mathrm{n} /+$ $/ \mathrm{I} /$ sequence as well as control items extra ransom and extra napkin involving cross-word vowel $+/ \mathrm{I} /$ or $/ \mathrm{n} /$; the data come from a larger study for which other stimuli were tested. Nine repetitions of each item were elicited in the carrier phrase That's a(n) produced at a normal speech rate.

We employed EPG, a method that tracks the contact of the tongue with an artificial palate, custommade for each participant (Gibbon \& Nicolaidis, 1999). Specifically, we used the WinEPG Articulate Instruments system (Wrench, Gibbon, McNeill, \& Wood, 2002), which records articulatory data at a sampling rate of $100 \mathrm{~Hz}$. The palates used with this system contain 62 built-in electrodes as shown in Figure 2 (a). These electrodes are schematically represented in Figure 2 (b), organized into eight columns and eight rows. 


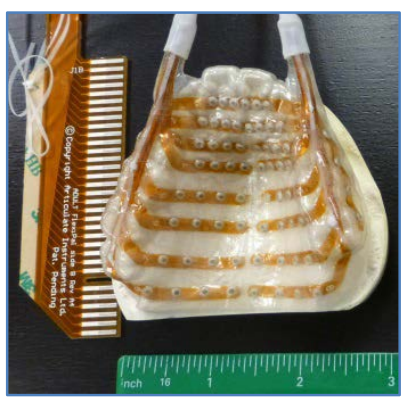

a.

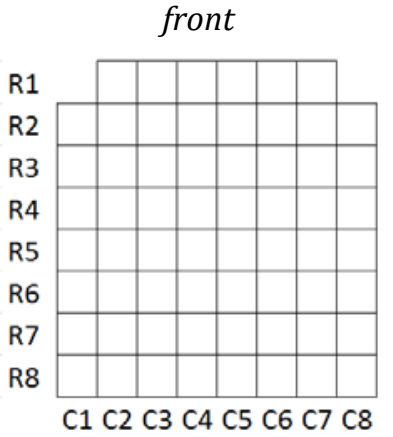

b.

Figure 2. (a) A sample EPG palate and (b) a schematic organization of 62 electrodes by column and row.

The data were analyzed both qualitatively and quantitatively; only the former analyses are presented here. These were based on visual examination of palate frames during the realization of both $/ \mathrm{n} / \mathrm{and} / \mathrm{x} /$. The consonants were classified in terms of place (alveolar versus post-alveolar) and stricture (stop versus approximant).

\section{Results}

3.1 L1 English patterns: Gradient assimilation We will begin with an examination of the control /n/+/x/ sequences produced by the Canadian English speakers. Figure 3 shows average contact profiles by consonant and speaker. The relative shading of the cells indicates the degree of contact between the tongue and the palate (dark purple $=100 \%$ ). Onset $/ \mathrm{n} /$ of napkin was typically produced with a closure at the alveolar ridge (the 3 anterior rows) and side contact; onset / $\mathrm{x} /$ of ransom was produced with a wide central opening and side contact in the post-alveolar and palatal regions of the palate (with the tongue tip/blade showing no contact given the approximant-like articulation). Of interest is the realization of coda / $\mathrm{n} / \mathrm{before}$ $/ \mathrm{x} /$ : while the consonant was still produced with an [n]-like anterior closure, its constriction tended to be more retracted, had less central anterior contact, and was overall more variable. This variability is indicative of a gradient (partial) retraction and opening of $/ \mathrm{n} /$ 's constriction in anticipation of $/ \mathrm{x} / \mathrm{as}$ can be seen in the sample temporal displays in Figure 4. The first image shows a relatively small degree of /n/retraction (speaker EN2), while the second image shows a considerably more retracted nasal (EN3). Such productions were typical of our L1 English speakers, indicative of a small (EN2) to moderate (EN1, EN3) overlap of the two gestures and the resulting gradient assimilation. These results are consistent with previous EPG studies of English that have shown typically gradient assimilation of coda /n/ to following consonants (Barry, 1991; Stephenson \& Harrington, 2002).

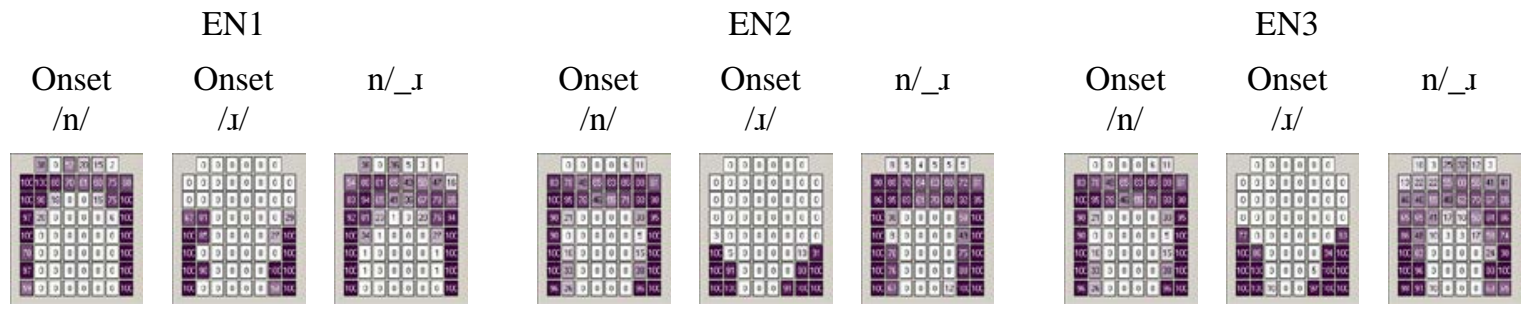

Figure 3. Average contact profiles by consonant (onset $/ \mathrm{n} /$, onset $/ \mathrm{x} /$, and coda $/ \mathrm{n} /$ before onset $/ \mathrm{x} /$ ) based on nine tokens for the L1 English control speakers. 


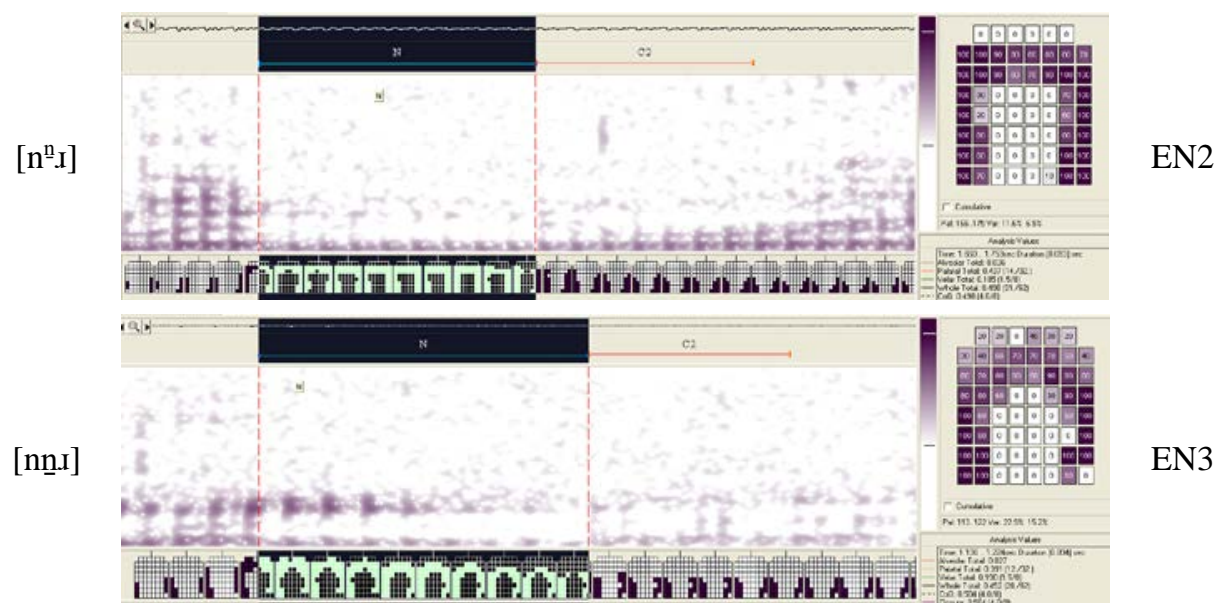

Figure 4. Sample temporal displays for the /n/+/x/ sequence for the L1 English speakers EN2 and EN3, with the nasal interval highlighted; palate frames taken every $10 \mathrm{~ms}$.

3.2 L2 English patterns: A range of observed strategies The results for the non-native speakers of English reveal that single onset $/ \mathrm{n} /$ and $/ \mathrm{I} /$ were overall produced in a target-like fashion - as an alveolar nasal stop and an approximant, respectively (with the exception of two alveolar tap tokens for JP2). The precise constriction location for the rhotic approximant was typically post-alveolar for all speakers except for the Spanish speakers who produced / $/ \mathrm{I} /$ in the alveolar region ([r] $]$ ), presumably adapting the native trill articulation. The overall approximant-like realization of the rhotic is noteworthy given the fact that L1 rhotics for these speakers are qualitatively different (see the Introduction). This is indicative of the (near)acquisition of the target consonants by our L2 speakers.

In contrast, the results were quite variable for the nasal+rhotic sequences. Some of the patterns observed in the speakers' productions were similar to those observed for the L1 controls, while other patterns were distinctly different. Six representative patterns of the L2 learners' productions are shown in Figure 5. The first image (a) illustrates the case of no overlap between $/ \mathrm{n} /$ and $/ \mathrm{I} /$, which were separated by a [ə]-like vocalic element. This pattern was found exclusively in the productions of the Japanese speakers JP1 and JP3. The second image (b) shows some small-scale gradient retraction of the nasal closure in anticipation of $/ \mathrm{x} /$. This is reminiscent of the L1 English pattern shown above for EN2, yet, the initial $/ \mathrm{n} /$ closure here was more anterior (dental $\left[n^{\mathrm{n}} \cdot \mathrm{I}\right]$ ). The third image (c) shows a more English-like pattern of gradient assimilation ([nn..]; see Figure 4). These two patterns were observed exclusively in the French speakers (FR1-FR3). The fourth image (d) shows categorical place assimilation of the nasal: the $/ \mathrm{n} /$ closure was strongly retracted to the post-alveolar/palatal region ([n. $x]$ ). Such productions were typical of speakers JP2 and SP1. The pattern seen in the fifth image (e) involves categorical assimilation of the nasal constriction in place accompanied by its assimilation in manner: the nasal was essentially identical in its

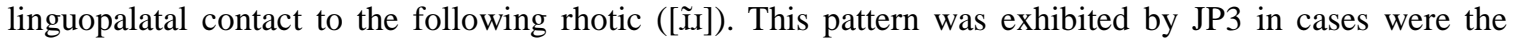
sequence was not broken up by an epenthetic vowel (a). Finally, the sixth example (f) shows bi-directional categorical assimilation: the nasal was assimilated in place (becoming post-alveolar), while the rhotic assimilated in stricture, becoming the affricate [ndr] $]$. This last pattern was typical for two Spanish speakers, SP2 and SP3. 
a.

b.

c. $[\mathrm{n} \underline{n} \mathrm{I}]$

d.

e.

f.

[n.I]

$[\tilde{\mathrm{I}} \mathrm{I}]$
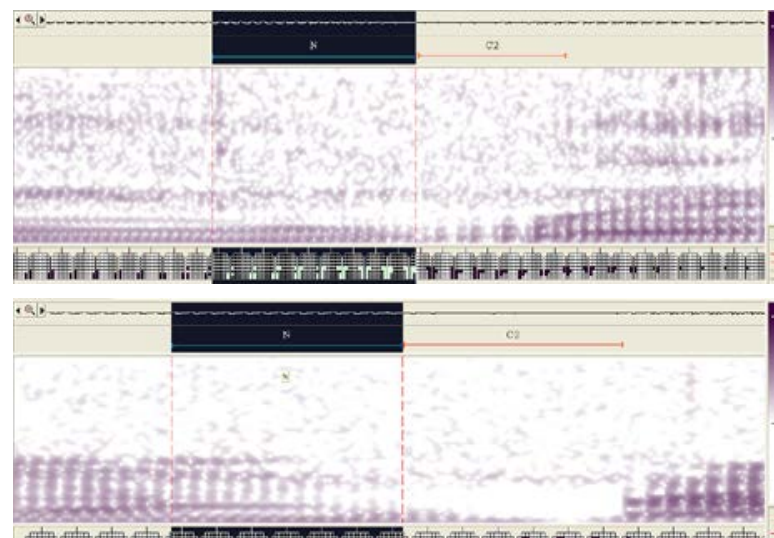

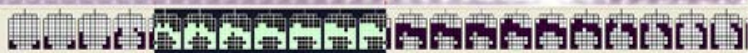

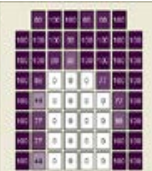

JP1
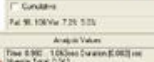

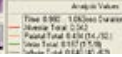

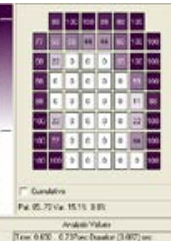

FR1

FR2

SP1

JP3

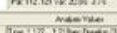

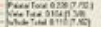

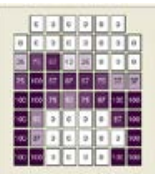

nen.

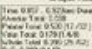

Figure 5. Representative patterns of temporal linguopalatal contact, with the nasal interval highlighted.

Overall, these patterns of coordination can be characterized as differing in the relative degree of gestural overlap and, as a consequence, in qualitative differences in assimilation patterns, ranging from no overlap due to epenthesis to strong overlap and categorical assimilation in place/stricture. These results are summarized in Table 1. 


\begin{tabular}{|c|c|c|c|c|c|c|}
\hline \multirow{2}{*}{$\begin{array}{c}\text { Realization } \\
\text { of } / n+d / \\
\text { sequence }\end{array}$} & \multicolumn{2}{|r|}{ Coordination patterns } & \multicolumn{4}{|c|}{ L1 group } \\
\hline & Overlap & Processes & English & French & Japanese & Spanish \\
\hline nəI & absent & epenthesis & & & JP1, JP3 & \\
\hline $\mathrm{n}^{\mathrm{n}} \mathrm{I} / \mathrm{n}_{\mathrm{n}}^{\mathrm{n}} \mathrm{I}$ & small & $\begin{array}{l}\text { limited gradient regressive place } \\
\text { assimilation (possible nasal fronting) }\end{array}$ & EN2 & $\begin{array}{l}\text { FR1, } \\
\text { FR3 }\end{array}$ & & \\
\hline nñ. & moderate & gradient regressive place assimilation & $\begin{array}{l}\text { EN1, } \\
\text { EN3 }\end{array}$ & FR2 & & \\
\hline$\underline{n} \mathrm{I}$ & large & $\begin{array}{l}\text { categorical regressive place } \\
\text { assimilation }\end{array}$ & & & JP2 & SP1 \\
\hline$\tilde{\mathrm{L} I}$ & large & $\begin{array}{l}\text { categorical regressive place } \& \\
\text { stricture assimilation }\end{array}$ & & & JP3 & \\
\hline$\underline{n}^{\mathrm{r}}{ }^{\mathrm{r}}$ & large & $\begin{array}{l}\text { categorical regressive place } \& \\
\text { progressive stricture assimilation }\end{array}$ & & & & $\begin{array}{l}\text { SP2, } \\
\text { SP3 }\end{array}$ \\
\hline
\end{tabular}

Table 1. Most frequently observed timing and assimilation patterns by speaker (two common patterns for JP3).

\section{Discussion}

The goal of this study was to determine whether L2 learners of English can acquire the target pattern of gradient regressive assimilation of the nasal to the following rhotic across word boundaries. This involves learning to produce both the sound of the target language and target-like temporal coordination of the consonant gestures. Previous articulatory phonetic studies have shown that nasal assimilation is typically categorical (and regressive) in Japanese and Spanish versus gradient in French (Honorof, 1999; Stephenson \& Harrington, 2002; Steele et al., to appear). Given this, continued L1-based articulatory influence was predicted to occur in the realization of $/ n+\mathrm{I} /$ sequences. Our results partly confirm this prediction. The French speakers' productions were overall most similar to the target patterns. This is expected given their typically gradient realization of nasal assimilation in their L1. The patterns shown by the Japanese and Spanish speakers were overall categorical in nature, but rather variable across speakers and not always consistent with the known L1 patterns. For example, across-word nasal+consonant sequences are not typically broken up by epenthetic vowels in Japanese, but are rather assimilated in place and stricture. The epenthesis observed here could reflect fluency issues (which also manifested itself in an overall slower speech rate, compared to other L2 speakers). In this respect, JP3's productions are particularly interesting, as this speaker produced some tokens with epenthesis and others with complete assimilation. The finding of bi-directional assimilation by two Spanish speakers is somewhat unexpected, but could be related to the incomplete acquisition of the English rhotic (realized as $[\underset{\tau}{\mathrm{r}}]$ ). It should be noted that previous studies have observed some post-nasal strengthening in assimilated sequences in L1 Spanish (Kochetov \& Colantoni, 2010; 2013). Further work is needed to compare L1 and L2 patterns of assimilation more closely.

To conclude, the results show that, although our learners (with the exception of our Spanish speakers) had essentially acquired the English rhotic articulation, they had not fully mastered the target C-C coordination patterns. This is consistent with findings of previous acoustic studies of L2 timing and coarticulation (Zsiga, 2003; Oh, 2008), highlighting the difficulty of acquiring gradient phonetic phenomena.

\section{References}

Barry, Martin C. 1991. Temporal modelling of gestures in articulatory assimilation. In Proceedings of the 12th International Congress of the Phonetic Sciences, vol. 4, 14-17. Aix-en-Provence: Université de Provence.

Browman, Catherine P., \& Goldstein, Louis. (1989). Articulatory gestures as phonological units. Phonology, 6, 201252.

Cebrian, Juli. (2000). Transferability and productivity of L1 rules in Catalan-English interlanguage. Studies in Second Language Acquisition, 22, 1-26.

Davidson, Lisa. (2010). Phonetic bases of similarities in cross-language production: Evidence from English and Catalan. Journal of Phonetics, 38, 272-288. 
Gafos, Adamantios. (2002). A grammar of gestural coordination. Natural Language and Linguistic Theory, 20, 269337.

Gibbon, Fiona, \& Nicolaidis, Katerina. (1999). Palatography. In William Hardcastle \& Nigel Hewlett (eds.), Coarticulation: Data, theory and techniques (pp. 229-245). Cambridge: Cambridge University Press.

Honorof, Douglas. (1999). Articulatory gestures and Spanish nasal assimilation. Ph.D. dissertation, Yale University.

Kochetov, Alexei. (2014). Japanese nasal place/stricture assimilation: Electropalatographic evidence. Poster presented at the 14th Conference on Laboratory Phonology, National Institute for Japanese Linguistics (NINJAL), Tokyo, Japan, July 2014.

Kochetov, Alexei \& Colantoni, Laura. (2010). Spanish nasal assimilation revisited: A cross-dialect electropalatographic study. Laboratory Phonology, 2, 487-523.

Kochetov, Alexei \& Colantoni, Laura. (2013). An electropalatography (EPG) study of nasal-trill/lateral sequences in Spanish. Proceedings of Meetings on Acoustics, 19. 9 pp.

Oh, Eunjin. (2008). Coarticulation in non-native speakers of English and French: An acoustic study. Journal of Phonetics, 36, 361-384.

Quilis, Antonio. (1993). Tratado de fonética y fonología españolas. Madrid: Gredos.

Ramsammy, Michael. (2011). The realization of coda nasals in Spanish. PhD thesis, University of Manchester.

Steele, Jeffrey, Colantoni, Laura, \& Kochetov, Alexei. (to appear). Gradient assimilation in French cross-word /n/+velar stop sequences. Journal of International Phonetic Association. 27 pp.

Stephenson, Lisa S., \& Harrington, Jonathan. (2002). Assimilation of place of articulation: Evidence from English and Japanese. Proceedings of the 9th AICSST, 592-597.

Tranel, Bernard. (1987). The sounds of French. Cambridge: Cambridge University Press.

Vance, Timothy J. (1987). An introduction to Japanese phonology. New York, N.Y.: SUNY Press.

Wrench, Alan, Gibbon, Fiona, McNeill, Alison M., \& Wood, Sara. (2002). An EPG therapy protocol for remediation and assessment of articulation disorders. In John H. L. Hansen \& Brian Pellom (eds.), Proceedings of the 7th International Conference on Spoken Language Processing (pp. 965-968). Denver, CO.

Zsiga, Elizabeth C. (2003). Articulatory timing in a second language: Evidence from Russian and English. Studies in Second Language Acquisition, 25, 399-432. 